\title{
Da implantação à implementação de ambientes virtuais tridimensionais: caminhos em construção
}

\author{
Elizabeth M. Rocha $^{1}$, Luana O. Elias ${ }^{1}$, Marcos K. Obara ${ }^{1}$, Marcelo Y. Hasegawa ${ }^{1}$, \\ Leonardo A. Neto ${ }^{1}$, Fabio Henrique N. Abe ${ }^{1}$ \\ ${ }^{1}$ Educação a Distância - Universidade Federal da Grande Dourados (UFGD) \\ Caixa Postal 322 - CEP: 79.825-070 - Dourados - MS - Brasil \\ elizabethrocha@ufgd.edu.br, \{luanaelias10, oba.marcos, marcelomyh1011, \\ leonardoaquinoneto, fabio.henrique.abe\} @gmail.com
}

\begin{abstract}
This article shows the main computational difficulties that limit the incorporation of Three Dimensional Virtual Learning Environments (3D VLE) in the educational component. This project uses the open-source proposal for the development of our $3 D$ environment with OpenSimulator. We conclude that the computational limit, though complex, can be overcome and the four experiments held by this project showed that the three-dimensional virtual space can be successfully applied to the academic proposal of distance courses, since the academics who study through the Web naturally access their virtual rooms, in this project's case, Moodle.
\end{abstract}

Resumo. Este artigo mostra as principais dificuldades computacionais que limitam a incorporação de Ambientes Virtuais Tridimensionais (AVA 3D) no componente educacional. O projeto utiliza a proposta open-source para o desenvolvimento de ambiente 3D, a partir do OpenSimulator. Conclui-se que a limitação computacional, embora complexa, pode ser superada e os quatro experimentos realizados decorrentes do projeto AVA $3 D$ mostram que o espaço virtual tridimensional pode se aplicar com êxito à proposta acadêmica de cursos a distância, uma vez que os acadêmicos que estudam pela Web acessam naturalmente suas salas virtuais, no caso deste projeto, o Moodle.

\section{Introdução}

Que tipos de interações e necessidades educacionais, comunicacionais e informacionais precisam ser adequadas de modo a permitir que professores e alunos, sob a forma de avatares, desenvolvam situações de estudo em mundos virtuais tridimensionais (AVA3D)? Neste artigo, portanto, tem-se a oportunidade de conferir as principais dificuldades computacionais que limitam o uso dessa tecnologia na educação.

Essa questão norteadora está sendo levantada no início do artigo para mostrar que, embora seja forte a tendência do uso virtual tridimensional em diversos aspectos do cotidiano da sociedade do século XXI, como o cinema, games e sites, o uso dessa tecnologia na educação tem tido um respaldo ainda insuficiente [Mattar, 2010].

Dentro do mundo digital do Second Life, "segunda vida", ambiente 3D criado pelo físico americano Phillip Rosendale em conjunto com a empresa Linden Lab, as pessoas têm a possibilidade de, na forma de avatar, expandir sua natureza, sua personalidade, pois são elas quem constroem este mundo [Dalfovo et al., 2008]. 
Segundo Wiziack (2007), a população no início de 2007 dentro do Second Life era de 2,8 milhões de habitantes, dos quais, de acordo com o relatório divulgado pela Linden Lab, 48.023 eram avatares brasileiros, o equivalente a 8,55\% do total, contra 149.055 (26,55\%) norte-americanos. Atrás do Brasil estão, nesta ordem, Japão, Alemanha, Reino Unido, França, Itália e Holanda.

O conceito do Second Life ultrapassa a barreira de ser somente um jogo eletrônico. Ele é considerado como um ambiente virtual tridimensional que tem o seu foco na interação entre usuários de diversos locais. Metaversos, como são chamados os ambientes proporcionados por determinados softwares, sofrem constantes modificações de acordo com o ambiente em que são inseridos. No caso específico desta pesquisa, a ideia é proporcionar essa interatividade através da reprodução do espaço físico da faculdade ao qual os alunos estão acostumados, para tornar mais interativa a relação entre professor e aluno e o ensino como um todo.

A proposta open-source em atendimento ao desenvolvimento de plataforma 3D se materializa a partir do OpenSimulator, ou simplesmente OpenSim, que, diferentemente do Second Life, é um projeto de código aberto, sob a licença BSD (Berkeley Software Distribution). A proposta foi criada em janeiro de 2007 para ser uma plataforma flexível e modular, favorável à criação de mundos virtuais personalizados, dando a oportunidade de qualquer pessoa ter permissão para copiar, modificar e usar o OpenSim com o propósito de atender as suas necessidades [Freire et al., 2010]. Embora o projeto esteja trabalhando com o OpenSim, por várias limitações identificadas, têm-se buscado ambientes 3D alternativos que garantam mais eficiência e qualidade, como o Unity, apresentado na seção 5.

A pesquisa de Dalgarno et al. (2011) mostra o forte interesse de pesquisadores na incorporação de mundos virtuais 3D no componente educacional, sobretudo em países como Austrália e Nova Zelândia. Essas pesquisas se vinculam à formação docente [Campbell, 2009; Gamage, Tretiakov; Crump, 2009], a percepções dos estudantes [Gregory; Masters, 2012] e a práticas didático-pedagógicas [Willems, 2009]. As pesquisas apontam que, embora sejam constatadas inciativas de ensino nessa direção, são fortes os obstáculos enfrentados para o uso dessa tecnologia. Mas em que direções apontam as dificuldades para uso da tecnologia virtual 3D?

Para responder os questionamentos levantados, este artigo apresenta elementos da literatura e experimentações produzidas sobre o assunto nos últimos anos, para, em seguida, apontar e discutir as limitações identificadas no que tange à implantação do AVA 3D.

\section{Uso da tecnologia virtual 3D no mundo e no Brasil}

$\mathrm{Na}$ publicação, em 2007, do informativo The Horizon Report, identificaram-se inciativas emergentes vinculadas ao uso de mundos virtuais e de Massively Multiplayer Online Games (MMOGs), com a perspectiva de que tais ações impactassem o cenário educacional em até cinco anos. Isso não só não aconteceu, como o uso dessa tecnologia na sala de aula virtual ou presencial ainda é praticamente inexistente, justamente pelo caráter desafiador da proposta, seja do ponto de vista cultural, seja pelas fragilidades computacionais, destacadas no item 4 deste trabalho, no que tange às plataformas do Second Life e do OpenSimulator. Os três anos desta pesquisa evidenciam a necessidade 
de mais investigação sobre as formas de melhoria de desenvolvimento para a viabilização da tecnologia 3D no espaço acadêmico e, mesmo, escolar [Dalgarno et al., 2011].

De um modo ou de outro, o certo é que as propostas de uso de AVAs 3D visam à ampliação e diversificação das formas de estudo, sob o viés da colaboração e da interação, seja para realização das atividades de estudo, seja para dar coesão social aos alunos na hora das atividades (Figura 01), seja para dar aos alunos certo poder sobre seu próprio aprendizado, na tentativa de ajudá-los a mudar seu modelo mental, quando a ação é estudar.

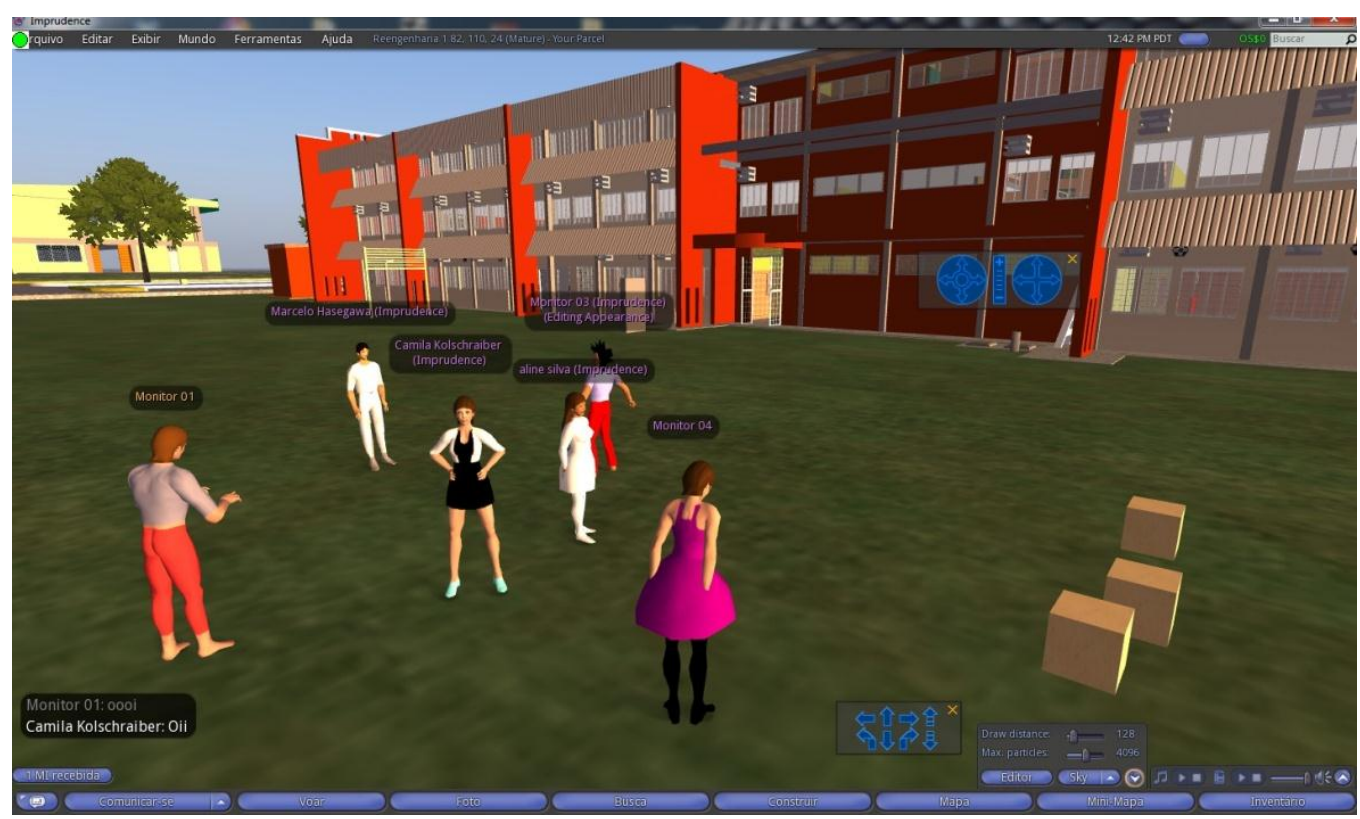

Figura 01 - Interação entre acadêmicos

Com iniciativas de pesquisas em ambientes virtuais 3D [Mattar; \&Valente, 2007] que retratam a necessidade de parcerias entre instituições de ensino da educação superior ou básica, os resultados advindos de pesquisas com uso dessa tecnologia apontam questões interessantes a uma revisão e atualização de proposta de mediação pedagógica e design didático no ensino presencial e a distância de cursos de graduação e pós-graduação.

Na leitura de Campos (2004), evidencia-se que, na disciplina de Astronomia, com o uso de modelos 3D de fenômenos astronômicos relativos à rotação da Terra em torno do seu eixo e do Sol e de ocorrências de eclipses do Sol e da Lua, usando um software de edição VRML, os alunos de graduação mostraram-se motivados para o estudo, além de mais interativos e cooperativos entre si, com maior desempenho da aprendizagem do conteúdo em questão, se comparados à outra turma-controle participante da pesquisa. Iniciativas de interação virtual em plataformas 3D também podem ser conferidas por algumas instituições particulares, como na proposta disponibilizada no endereço http://connepi.ifal.edu.br/.

Ora, se participar de estudos, como em ambientes virtuais $3 \mathrm{D}$, proporciona ao 
estudante um modo e estilo diferente de estimular sua ação cognitiva, pois aciona seu campo motivacional, é imprescindível investigar mais a fundo como se constrói e se viabiliza essa tecnologia no meio educacional. A pesquisa apresentada neste artigo tomou como caminho e ponto de partida o meio universitário. Afinal, espera-se que o espaço acadêmico favoreça campos de aprendizagem diferenciados de modo que possam adquirir habilidades compatíveis com as necessidades do século XXI, como trabalho em equipe, rápidas, eficientes e múltiplas formas comunicacionais.

\section{A implantação e a implementação do OpenSim: caminhos virtuais em construção}

Esta pesquisa foi iniciada em 2010 em uma universidade pública federal situada em Dourados (MS), pelo núcleo de Educação a Distância, e teve como objetivo central mapear e analisar o processo das construções cognitivas de professores e estudantes no ambiente 3D. O prazo estabelecido pelo triênio de 2010 a 2012 para realização do projeto se mostrou insuficiente para o alcance pleno desse objetivo, por dois motivos principais. O primeiro, pela complexidade da implantação da tecnologia 3D, no que confere à limitação na modelagem de objetos, visualizador, integração com o Moodle e acessibilidade. $\mathrm{O}$ segundo, pelo desconhecimento dos professores em elaborar atividades didáticas em ambientes dessa natureza. Isso evidenciou a limitação da base cultural dos professores para lidar com essa tecnologia como suporte didático.

A memória da trajetória da proposta exige dizer que, em 2010, nascia, junto com a pesquisa 3D, o setor de Educação a Distância da universidade. Durante a implementação do projeto, percebeu-se que o impacto inicial das implicações desse projeto na comunidade acadêmica da $\mathrm{EaD}$ foi a constatação do quanto a tecnologia virtual 3D é, ainda, bem desconhecida, por desenvolvedores daquela região. Esse fator foi determinante no tempo destinado à implantação do projeto, que, previsto para um ano, levou todo o triênio. Na prática, tem-se, de forma experimental, uso do AVA 3D no setor de EaD da universidade. A implementação ainda está em construção, ou seja, embora a tecnologia esteja incorporada no setor de EaD, sua proposta vinculada aos cursos de graduação a distância, que, atualmente, são os de Licenciatura em Computação e Licenciatura em Pedagogia, para média de 250 acadêmicos, ainda não está suficientemente disponível.

A implantação do projeto foi dividida em três fases. Na primeira, procedeu-se a ampla revisão bibliográfica; na segunda, foi feita a escolha da plataforma, optando-se, inicialmente, pela OpenSimulator, por ser livre, intuitiva e gratuita. Na terceira fase efetuou-se a integração do AVA Moodle com o OpenSim através do módulo Sloodle [Nakamura, 2008]. A última fase contemplou, ainda, a realização de quatro testespiloto.

Em 2011, o projeto realizou duas experiências-piloto, de 120 min cada. Na primeira, o público foi de 18 alunos da disciplina de Informática na Matemática. Na segunda, o público foi de 10 professores de Matemática da rede pública e particular do Mato Grosso do Sul. Em 2012, o projeto realizou mais dois testes experimentais, envolvendo 10 acadêmicos do curso de Licenciatura Plena em Matemática.

Em todos os testes, os participantes assumiram, o tempo inteiro, o papel de agentes ativos na realização individual e coletiva das atividades propostas. As atividades consistiam em criar o avatar pessoal (ação individual), modelar um objeto (ação 
individual) e resolver as atividades solicitadas, conforme a Figura 02.

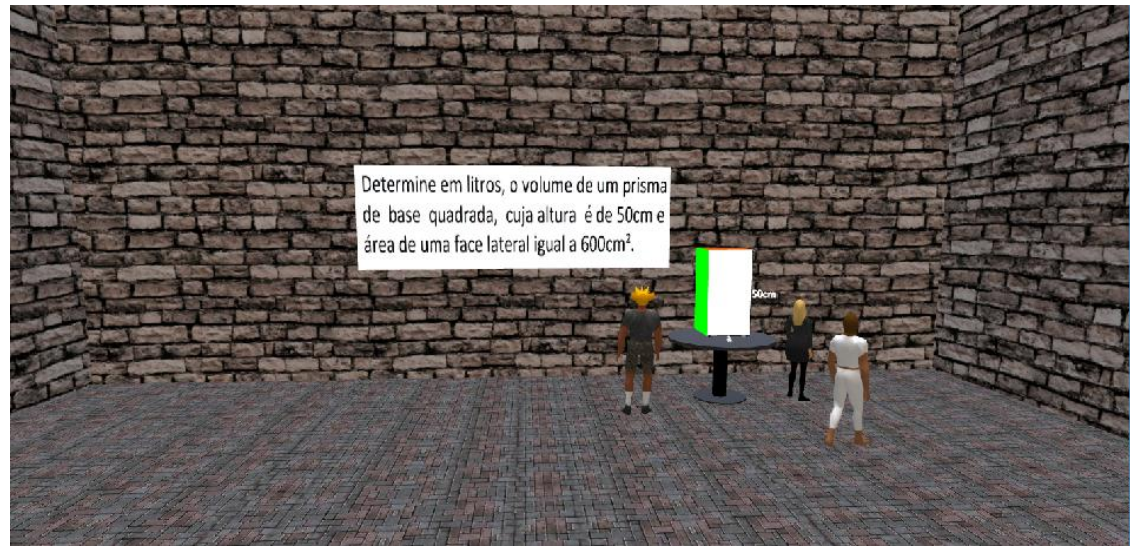

Figura 02 - Acadêmicos em interação letiva

As atividades afins e convergentes relativas aos testes-piloto visaram dar autonomia aos participantes no uso da plataforma, a partir da execução de seis etapas para concluir a proposta de experimentação. Essas etapas incluíram: instalação do programa de gravação e do visualizador e criação do avatar através do Moodle (individual); personalização do avatar (individual); visita ao campus virtual da universidade (individual); realização dos desafios matemáticos no ambiente (em grupo); construção de objetos (individual); entrevista sobre a experimentação (em grupo).

Os sentimentos foram diversos, estampados nas filmagens dos grupos, revelando fisionomias risonhas, o resgate do espírito lúdico (quando um aluno corria, virtualmente, atrás do outro, quando "chutava" ou "empurrava" no sentido de descontração) e, ainda, a dificuldade de trabalhar em grupo, quando seus semblantes ficaram mais sérios e seus corpos denotavam desconforto ao levantar e esticar braços. As atividades propostas, de fato, os colocaram em situação de ação e reflexão.

Apesar de o ambiente ser um simulador de mundos, há diversas mensagens explicativas em forma de textos, pela limitação de situações didáticas automatizadas, na forma de agentes inteligentes capazes de comunicar-se automaticamente com o alunoavatar, como no caso de chatterbots, na perspectiva do trabalho de Alencar e Magalhães Neto (2010). A análise dos vídeos da experimentação mostrou dificuldades na realização das atividades, não pelo caráter de manipulação do avatar, ou mesmo pela modelagem dos objetos, mas pela dificuldade de leitura, interpretação e capacidade de analogia com a atividade proposta.

\section{Principais dificuldades de percurso na implantação do projeto}

A pesquisa AVA 3D evidenciou dificuldades de percurso relacionadas mais efetivamente à área computacional, considerando que não houve propostas didáticas suficientes no projeto, na forma de cursos, por exemplo, que ajudem a extrair elementos mais norteadores quanto às dificuldades educacionais, no que confere à formação docente. A implantação de ambiente imersivo, com os participantes na forma de avatares, interagindo de forma integrada, articulada e em tempo real no mundo virtual, torna-se mais complexa do que propostas que ofertam ao usuário visitação em mundos 
3D, de forma visual, interagindo pontualmente com os objetos, na forma de passeio virtual, como Projeto Missões e Brasília 3D, apontados no trabalho de Antoniazzi (2009), que trata de simulação computacional de ambientes históricos.

Durante a implantação do AVA 3D desta pesquisa, foram utilizadas as ferramentas disponíveis no OpenSimulator; porém, a necessidade de objetos mais detalhados condicionou a busca de uma ferramenta mais robusta de modelagem 3D. Optou-se, então, pela utilização do Blender, software gratuito e de código aberto, que permite a criação de objetos mais definidos, mais leves e com um visual mais real.

O OpenSimulator possui limitação na modelagem de objetos detalhados, pela restrição apenas às formas primitivas cubo, cilindro, esfera e tórus. Em contrapartida, o Blender permite a criação de qualquer forma que a criatividade permitir. Os objetos criados através da ferramenta Blender podem ser exportados e utilizados dentro do OpenSimulator.

O visualizador Imprudence, inicialmente adotado pelo projeto, não nos supriu com todas as necessidades. Por exemplo, na forma de importação de objetos criados no Blender, só suportava o método Targa de conversão, que corresponde à transformação de modelo tridimensional em uma imagem bidimensional, com padrão Targa de mapeamento, diferenciando profundidade, largura e altura pelas cores vermelho, azul e verde, e suas intensidades, deformando, contudo, os objetos no momento da descompressão. A superação dessa dificuldade foi a substituição do Imprudence pelo visualizador Exodus, que possibilita a importação de objetos no formato Collada, compressão que mantém os padrões tridimensionais do objeto, com garantia de qualidade, sem distorções.

As opções de execução do OpenSimulator são Standalone e Grid, cada uma direcionada a uma utilização especifica. Neste projeto, foi adotada a utilização do OpenSimulator no modo Grid, para ter-se controle detalhado de quem acessa as regiões, de modo a evitar travamentos do servidor: caso trave, somente uma região ficará inacessível, diminuindo o impacto de um travamento, além de possibilitar a visualização exata do tráfego de dados e a utilização dos processadores de cada região individualmente.

Outra forte dificuldade computacional consiste em tornar o ambiente acessível a todos no que diz respeito a qualquer tipo de máquina, seja ela desktop ou notebook, de última geração ou um pouco mais ultrapassado, para que todos possam ter acesso a esse AVA 3D, sem encontrar dificuldades em suas máquinas.

No que confere às limitações no campo educacional, as poucas experimentações com participantes da pesquisa apontam que, apesar de a educação seguir dentro de uma evolução, na qual encontram-se propostas de ensino com base em uso de softwares educativos em computador e meios eletrônicos, existe forte resistência por parte de professores e acadêmicos em entender e se adequar a situações imersivas em mundos virtuais [Fonseca, 2008].

Os testes realizados com os acadêmicos mostraram que a plataforma responde bem com até 20 pessoas simultâneas. Essa informação é compatível com o informe do site http://simhost.com. No entanto, a plataforma utilizada no projeto, a OpenSimulator, apresenta muitos problemas de instabilidade dos servidores, fazendo com que ocorram frequentes perdas de scripts e objetos. De acordo com o site oficial do OpenSimulator, 
esses problemas são decorrentes do alto tráfego de dados, pois o servidor, ao enviar dados, espera o visualizador retornar com o que foi pedido; o visualizador, por sua vez, tem um tempo para retornar estes dados; quando o tráfego é muito intenso, o tempo expira e acontece a perda de conexão.

Outra questão importante é que a velocidade de Internet necessária para o usuário está entre $500 \mathrm{Kbps}$ e $2 \mathrm{Mbps}$. A velocidade de $2 \mathrm{Mbps}$ é suficiente para carregar as estruturas e texturas em uma velocidade boa; a velocidade de 500Kbps é o mínimo para uma experiência agradável, mas com uma espera maior. Uma Internet mais lenta também acessa normalmente o ambiente, mas será uma experiência estressante devido à demora para carregar os objetos e texturas.

\section{5. $O$ estado do devir: Unity, como possível solução computacional à proposta AVA 3D?}

Diante das limitações apresentadas pela plataforma OpenSimulator, tem-se apostado no Unity, como proposta alternativa em estudo. O Unity foi fundado em 2004 por David Helgason (CEO), Francisco Nicolau (CCO), e Joachim Ante (CTO), em Copenhague, na Dinamarca.

$\mathrm{O}$ foco dessa engine é democratizar o desenvolvimento de jogos e tornar a criação de ambientes em $2 \mathrm{D}$ e $3 \mathrm{D}$, um conteúdo interativo para todos os usuários. Neste contexto, a equipe do Unity desenvolveu um protótipo de um jogo online, onde é possível autenticar um usuário e dar um nome a um avatar, que neste caso é representado por um "cubo", neste protótipo, que funciona tanto para web, quanto para desktop, é possível notar que as qualidades gráficas são de melhor qualidade se comparadas à plataforma do OS, conforme destaque, em termos de nitidez, de forma comparativa nas figuras 03 e 04 :

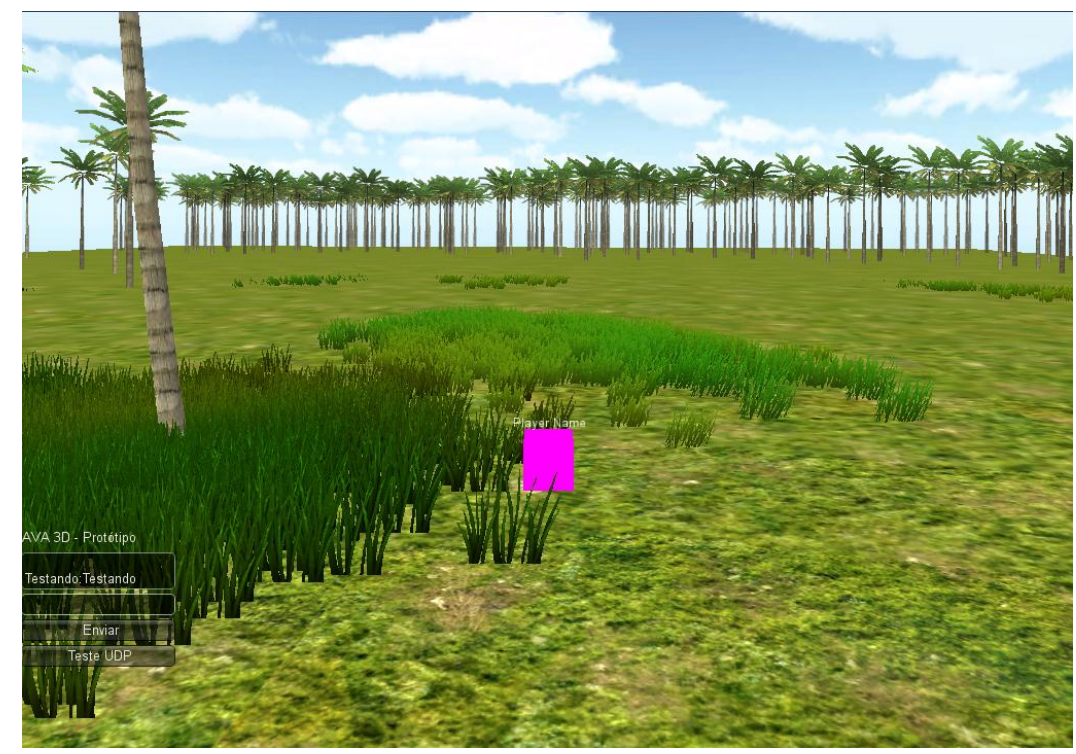

Figura 03 - Unity3D - Protótipo de um jogo online 


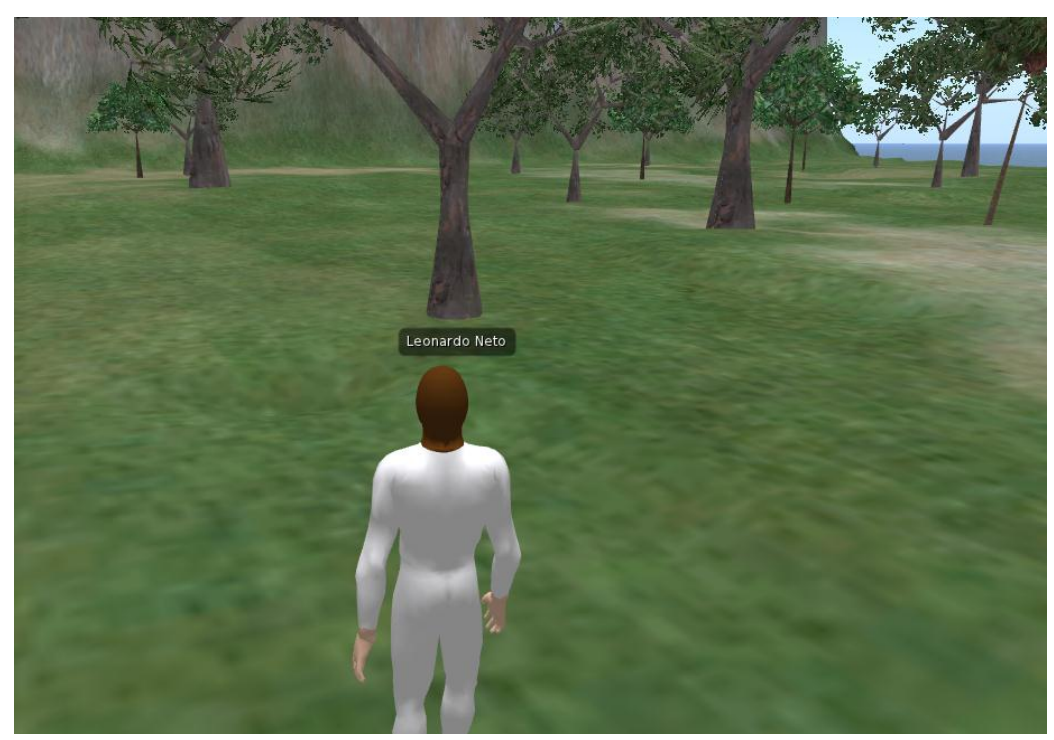

Figura 04 - OpenSim

O Unity, ainda, é mais leve e possibilita o acesso ao AVA3D diretamente do browser, apenas necessitando da instalação de um plugin e baixando as regiões somente na primeira vez que entrar na plataforma, tendo também a opção de baixar o arquivo do jogo para o computador e executar sem a necessidade do browser. Embora não seja ferramenta opensource, oferece a versão gratuita para projetos sem fins lucrativos.

Apesar de todas estas funcionalidades estarem presentes na versão gratuita do software, há ainda a opção de utilizar a versão pro do Unity, que, ao ser adquirida, habilita a função de exportar o jogo para celulares e tablets que utilizam os sistemas $i O S$ e Android. Com essa versão, também existe a opção de dar acesso a videogames, como Playstation 3, XBOX 360 e Nintendo Wii.

\section{Considerações finais}

Será que acadêmicos que já estudam e professores que já ensinam em espaços virtuais assimilam melhor a cultura do espaço 3D? Essa pergunta, aliada à pergunta inicial deste artigo, ainda carece de respostas mais consistentes, considerando que há real dificuldade computacional para uso de AVA 3D, sobretudo em se tratando de ferramentas open source.

Embora se admita que a limitação computacional da plataforma OpenSimulator limite, também, a aplicação educacional e ação pedagógica, os quatro experimentos realizados decorrentes do projeto AVA 3D mostram que o espaço virtual tridimensional pode se aplicar com êxito à proposta acadêmica de cursos a distância, uma vez que os acadêmicos que estudam pela Web acessam naturalmente suas salas virtuais, no caso deste projeto, o Moodle. Trata-se de uma proposta arrojada e, certamente, complexa de ser alcançada. Há que se considerar, ainda, estudos que contemplem o uso de técnicas de tutores inteligentes dentro da perspectiva de Inteligência Artificial (IA), com o intuito de tornar ainda mais eficiente a proposta pedagógica.

No caso desta pesquisa, a implantação da proposta levou três anos. Como 
continuidade da proposta, pretende-se, a partir do segundo semestre de 2013, em caráter de implementação, aplicar, gradativamente, situações didáticas vinculadas às disciplinas dos cursos de graduação a distância. Ainda no primeiro semestre de 2013, aos professores, por adesão, será oferecido um curso de capacitação, pela equipe do projeto, para criação de atividades didáticas para AVA 3D. A ideia é que o acadêmico, a partir da sua sala pelo Moodle, tenha acesso a um link que o redirecione à atividade na plataforma virtual 3D, ampliando, portanto, suas interações letivas.

Desse modo, professores e acadêmicos terão oportunidade de ampliar suas ações cognitivas. Os professores receberão estímulos no sentido de pensar situações didáticas adaptadas a simuladores virtuais; e os acadêmicos receberão orientações no sentido de ampliar suas possibilidades de estudo.

A conclusão desta pesquisa aponta questionamentos justamente para mostrar que se trata de uma proposta de estudo recente, com poucas experimentações, sobretudo pela dificuldade computacional e, portanto, com amplo campo para realização de estudos que ajudem a nortear caminhos e tendências para uso da tecnologia virtual 3D nas aulas.

\section{Referências}

Alencar, M. A. S. e Magalhães Netto, J. F. (2010) "CyberPoty: Um Chatterbot 3D para Interação com usuários de um Portal de Educação a Distância", http://www.brie.org/WIE2010/pdf/sp02_05.pdf. Acesso em: 29/09/2012.

Alves, L. e Brito, M. (2005) "O Ambiente Moodle como Apoio ao Ensino Presencial", http://www.abed.org.br/congresso2005/por/pdf/085tcc3.pdf, Acesso em: 15/03/2012.

Antoniazzi, A. (2009) Simulação computacional de ambientes históricos: procedimentos metodológicos para estudo de caso na Praça Dante Alighieri e no entorno imediato. Dissertação apresentada ao Programa de Pós-Graduação em Arquitetura da Faculdade de Arquitetura da Universidade Federal do Rio Grande do Sul. Disponível em http://www.lume.ufrgs.br/handle/10183/16934. Acesso em 12/09/2012.

Campos, J. A. S. de (2004). Um estudo exploratório sobre uso de ambientes virtuais não imersivos em 3D no ensino de Astronomia. Dissertação apresentada ao Mestrado em Informática do Instituto de Matemática/Núcleo de Computação da Universidade Federal do Rio de Janeiro. Disponível em: http://www.nce.ufrj.br/ginape/publicacoes/dissertacoes/d_2004/d_2004_jose_adolfo_ snajdauf_de_campos.pdf. Acesso em: 18/03/2012.

Campbell, M. (2009) "Using 3D-virtual worlds to teach decision-making”, Proceedings $\begin{array}{ll}\text { ascilite } \quad \text { Auckland 2009, } & \text {, }\end{array}$ http://www.ascilite.org.au/conferences/auckland09/procs/campbell-m.pdf. Acesso em 18/08/2012.

Dalgarno, B. et al. (2011) "Institutional support for and barriers to the use of 3D immersive virtual worlds in higher education", http://www.leishmanassociates.com.au/ascilite2011/downloads/papers/Dalgarno-full.pdf. Acesso em 
$18 / 08 / 2012$.

Dalfovo, O. et al. (2008) "Sistemas de informação: Ambiente Second Life Um Sistema Como Ferramenta de Marketing”, http://www.convibra.com.br/2008/artigos/242_0.pdf. Acesso em: 15/03/2012.

Fonseca, M. G. (2008) "A tecnologia em favor da educação", http://www.edumat.com.br/wp-content/uploads/2008/12/a-tecnologia-em-favor-daeducacao.pdf. Acesso em: 14/03/2012.

Freire, A., Rolim, C. e Bessa, W. (2010) "Criação de um Ambiente Virtual de EnsinoAprendizagem usando a plataforma OpenSimulator", http://connepi.ifal.edu.br/ocs/index.php/connepi/CONNEPI2010/paper/viewFile/684/ 410, Acesso em: 20/08/2011.

Gamage, V., Tretiakov, A. and Crump, B. (2009) "Educators' perceptions about using MUVE for teaching", Proceedings ascilite Auckland, 2009, http://www.ascilite.org.au/conferences/auckland09/procs/gamage-poster.pdf. Acesso em 17/08/2012.

Gregory, S. and Masters, Y. (2012) "Real thinking with virtual hats: A role-playing activity for pre-service teachers in Second Life". Australasian Journal of Educational Technology 2012, 28 (Special issue, 3), pages 420-440, http://www.ascilite.org.au/ajet/ajet28/gregory.html. Acesso em 17/08/2012.

Mattar, J.; Valente, C. (2007), Second Life e Web 2.0 na educação: o potencial revolucionário das novas tecnologias, São Paulo: Novatec.

Mattar, J. (2010), Games em Educação: como os nativos digitais aprendem, São Paulo: Pearson Prentice Hall.

Nakamura, R. (2008), Moodle: como criar um curso usando a plataforma de Ensino à Distância, São Paulo: Farol do Forte,http://cead.unifalmg.edu.br/download/livromoodle.pdf, Acesso em: 03/04/2012.

New Media Consortium. (2007) NMC Horizon Report 2007 Edition. Austin: New Media Consortium, http://www.nmc.org/pdf/2007_Horizon_Report.pdf. Acesso em: 25/09/2012.

Willems, J. (2009) "Different spaces but same places: Possibilities, pitfalls and persistent practices in Second Life", Proceedings ascilite Auckland 2009, http://www.ascilite.org.au/conferences/auckland09/procs/willems.pdf. Acesso em $17 / 08 / 2012$.

Wiziack, J. (2007) "A segunda vida - por que milhões de pessoas embarcam no mundo de fantasia e lucros do Second Life”, Revista IstoÉ, n. 1948, 28 fev. 2007. 